\title{
Article \\ Current Status of Genetic Diagnosis Laboratories and Frequency of Genetic Variants Associated with Cystic Fibrosis through a Newborn-Screening Program in Turkey
}

\author{
Sevcan Tug Bozdogan 1,2, Cem Mujde ${ }^{2}$, Ibrahim Boga ${ }^{1,2}$, Ozge Sonmezler ${ }^{2}$, Abdullah Hanta ${ }^{2} \oplus$, \\ Cagla Rencuzogullari ${ }^{2}$, Dilek Ozcan ${ }^{3}$, Derya Ufuk Altintas ${ }^{3}$ and Atil Bisgin ${ }^{1,2, *}$ (1) \\ 1 Medical Genetics Department, Faculty of Medicine, Cukurova University, Adana 01250, Turkey; \\ sevcantb@gmail.com (S.T.B.); ibr.boga@gmail.com (I.B.) \\ 2 AGENTEM (Adana Genetic Diseases Diagnosis and Treatment Center), Cukurova University, \\ Adana 01250, Turkey; cemmujde@gmail.com (C.M.); ozgesonmezler@gmail.com (O.S.); \\ abdullahhanta@gmail.com (A.H.); caglaorucrencuz@gmail.com (C.R.) \\ 3 Faculty of Medicine, Department of Pediatric Allergy and Immunology Adana, Cukurova University, \\ Adana 01250, Turkey; dilekkaragoz1977@hotmail.com (D.O.); deryaufuk@gmail.com (D.U.A.) \\ * Correspondence: abisgin@yahoo.com; Tel.: +90-5325652167
}

Citation: Bozdogan, S.T.; Mujde, C.; Boga, I.; Sonmezler, O.; Hanta, A.; Rencuzogullari, C.; Ozcan, D.; Altintas, D.U.; Bisgin, A. Current Status of Genetic Diagnosis Laboratories and Frequency of Genetic Variants Associated with Cystic Fibrosis through a Newborn-Screening Program in Turkey. Genes 2021, 12, 206. https://doi.org/10.3390/ genes12020206

Academic Editor: Munis Dundar Received: 15 December 2020

Accepted: 27 January 2021

Published: 31 January 2021

Publisher's Note: MDPI stays neutral with regard to jurisdictional claims in published maps and institutional affiliations.

Copyright: (C) 2021 by the authors Licensee MDPI, Basel, Switzerland. This article is an open access article distributed under the terms and conditions of the Creative Commons Attribution (CC BY) license (https:// creativecommons.org/licenses/by/ $4.0 /)$.

\begin{abstract}
Background: Cystic fibrosis (CF) is the most common worldwide, life-shortening multisystem hereditary disease, with an autosomal recessive inheritance pattern caused by mutations in the cystic fibrosis transmembrane conductance regulator (CFTR) gene. The national newborn screening (NBS) program for CF has been initiated in Turkey since 2015. If the immunoreactive trypsinogen (IRT) is elevated (higher than $70 \mu \mathrm{g} / \mathrm{L}$ in the second control) and confirmed by sweat test or clinical findings, genetic testing is performed. The aims of this study are to emphasize the effect of NBS on the status of genetic diagnosis centers with the increasing numbers of molecular testing methods, and to determine the numbers and types of CFTR mutations in Turkey. Methods: The next-generation sequencing (NGS) and multiplex ligation-dependent probe amplification (MLPA) results of 1595 newborns, who were referred to Cukurova University Adana Genetic Diseases Diagnosis and Treatment Center (AGENTEM) for molecular genetic testing, were evaluated with positive CF NBS program results since 2017. Results: According to the results; 560 (35.1\%) of the 1595 patients carried at least 1 (one) CF-related variant, while 1035 patients (64.9\%) had no mutation. Compound heterozygosity for two mutations was the most common in patients, while two detected variants were homozygote in 14 patients. A total of 161 variants were detected in 561 patients with mutations. Fifteen novel variants that have not been previously reported were found. Moreover, p.L997F was identified as the most frequent pathogenic mutation that might affect the IRT measurements used for the NBS. The distribution of mutation frequencies in our study showed a difference from those previously reported; for example, the well-known p.F508del was the third most common ( $n=42$ alleles), rather than the first. The most striking finding is that 313 cases had a pathogenic variant together with the V470M variant, which might have a cumulative effect on CF perpetuation. Conclusion: This study is the first to determine the mutational spectrum of CFTR in correlation with the NBS program in the Turkish population. NBS for CF raises issues regarding screening in diverse populations, both medical and non-medical benefits, and carrier identification. Through the lens of NBS, we focused on the integrated diagnostic algorithms and their effect on the results of genetic testing.
\end{abstract}

Keywords: cystic fibrosis; newborn screening; genetic testing; population genetics

\section{Introduction}

Cystic fibrosis (CF) is the most common worldwide, life-shortening multisystem disease with an autosomal recessive inheritance pattern affecting 1 in 3300 to 1 in 4800 neonates and 1 in 2500 white individuals [1,2]. CF is a hereditary disease (MIM \#219700) 
caused by mutations in the cystic fibrosis transmembrane conductance regulator (CFTR) gene (MIM *602421) [3]. The cystic fibrosis gene, CFTR, was firstly identified in 1989, is located at the 7q13 position [4], and spans $\sim 190$ kilobases $(\mathrm{kb})$ on chromosome 7q31.2 with 27 exons. The CFTR gene encodes the CFTR protein that functions as a Cl-selective anion channel gated by cycles of ATP binding and hydrolysis at its nucleotide-binding domains [5]. Regarding CFTR gene mutations, to date, there are 1908 identified mutations, of which 982 are missense or nonsense [6], while the deletions and duplications of complete exons account for $1-2 \%$ of all mutations [7].

Mutations in the CFTR gene may result in defective protein processing that leads to changes in function and regulation of the chloride channel. CF affects exocrine glands; chloride secretion is diminished, sodium absorption and removal of water from secretions are increased through epithelial sodium channels, and the secretions are therefore abnormally viscous [8]. It mainly involves the lungs and pancreas, but also the upper airways, liver, intestine, and reproductive organs; $99 \%$ of the affected male patients are infertile due to obstructive azoospermia, and $87 \%$ of patients have exocrine pancreatic insufficiency. Its importance is due to the fact that it is a life-shortening disease, and the median predicted survival age and the median life expectancy are 47.4 and 44.4 years, respectively [9]. The longer patients with CF live, the worse their quality of life may become, in addition to the increased burden and cost of treatment [10]. Morbidity and mortality in CF are attributed most commonly to pulmonary disease, characterized by chronic lung infections and airway inflammation. Other common clinical manifestations are failure to thrive, pancreatic insufficiency, meconium ileus, and infertility resulting from a congenital bilateral absence of the vas deferens [1].

The national newborn screening (NBS) program for CF in Turkey has been in operation since 2015, and it was initiated by the Public Health Institution of Turkey. Immunoreactive trypsinogen (IRT) measurement as a first-tier testing method for newborns is used in NBS. Infants who are identified as positive in the NBS program are directed to CF centers for sweat testing followed by clinical assessment by pediatric allergy and immunology specialists. Then, the infants are referred to medical geneticists and genetic diagnosis centers for molecular genetic testing to also identify the mutational status and provide counseling to the family. Even though the screening tests have low false-positive rates, sweat testing and careful physical examination by pediatric immunologists are performed. As of 2017, genetic analyses via next-generation sequencing have been carried out at the main core center.

With this new NBS program, Turkey has taken a giant leap, screening a total of 1.2 million live births in 2019 and 1.25 million in the previous year according to the country's statistical institute (TUIK) [11]. For this NBS, the entire program's goal is to identify the disease and provide clinical answers to newborns. However, when screening via the IRT protocol, genetic diagnosis centers were required to overcome the large testing numbers and examine the data to identify connections with disease and variants, as research in this area is lacking in the Turkish population (excluding a single study from the IRT side with limited and early data [12]).

As a core center in our region, serving an area from south-east to the south-west of Turkey and dealing with all genetic diseases as well as CF molecular testing since its establishment, our aim within this study is, therefore, to identify how an NBS program for CF can affect the genetic diagnosis center's status with the increasing numbers of molecular testing methods, from the used techniques to the genotype variation of the CFTR gene. Moreover, in this study, we used the population-based globally well-known datasets, such as gnomAD and CFTR2, in order to assist genetic diagnostic laboratories and to demonstrate the analysis of large cross-population sequencing data in a large cohort that can significantly improve disease variant interpretation by assessing the variant frequency for our population with the varying prevalence. 


\section{Materials and Methods}

\subsection{Patients and Sampling}

Peripheral blood samples of 1595 newborns with positive CF NBS program results since April 2017 who were to Cukurova University Adana Genetic Diseases Diagnosis and Treatment Center (AGENTEM) referred for molecular genetic testing were included in this study. The CF newborn screening protocol in Turkey includes several steps, starting from sampling blood samples of newborns via sampling cards (Guthrie cards) at $72 \mathrm{~h}$ of life. Those whose first IRT level is higher than $\geq 90 \mu \mathrm{g} / \mathrm{L}$ were called for a second IRT measurement 7-14 days after birth. Then, if it is above $\geq 70 \mu \mathrm{g} / \mathrm{L}$, the infant was directed to the nearest $\mathrm{CF}$ center. The referred patients were evaluated at least once per three months, and the sweat test was performed for each infant with a gestational age of 38 weeks or more and a minimum weight of $2000 \mathrm{~g}$ together with clinical assessment. The NBS program's CF sweat testing results are considered to be abnormal when they are greater than $90 \mathrm{mmol} / \mathrm{L}$.

Informed parental consent was obtained for all patients in accordance with the ethical standards of the institutional ethical committee (Cukurova University Faculty of Medicine Non-Invasive Clinical Research Ethics Commission) and the Helsinki declaration.

Peripheral blood samples were collected for genomic DNA isolation via the QIAamp DNA Blood Mini Kit (Qiagen, Hilden, Germany), according to the manufacturer's instructions. The quality of DNA samples was assessed with a Qubit Fluorimeter (Thermo Fisher Scientific, Waltham, MA, USA).

\subsection{Next-Generation Sequencing (NGS)}

The next-generation sequencing workflow was performed to achieve a minimum of $300 \mathrm{X}$ coverage on an Illumina MiSeq (Foster City, CA, USA) platform via a custom-designed by our center's CFTR gene panel (QIAseq Targeted DNA Custom Panels-CDHS-12025Z65 , QIAgen, Hilden, Germany), including all exons, introns, and $1 \mathrm{~kb}$ of the $5^{\prime}$ promoter regions and the $3^{\prime}$ UTRs.

\subsection{Bioinformatics Analyses}

Quality control parameters were checked for both sequencing and variant qualities via the QIAGEN Clinical Insight (QCI) Analyze tool and the QCI Interpret interface. Total yield, sequencing quality score, depth of coverage, the quality score of variants, forward/reverse read balance, population, and variant frequencies were assessed as primary variant analysis. Variants were categorized based on their pathogenicity according to the American College of Medical Genetics (ACMG) criteria as pathogenic, likely pathogenic, variant of uncertain significance (VUS), likely benign, and benign. In silico analysis tools, including SIFT, B-SIFT, Polyphen-2, MutationTaster, BLOSUM, PROVEAN, CADD, DANN, GeneSplicer, PhyloP, MaxEntScan, and QCI Inferred Activation, were also used for the further examination of the VUSs.

\subsection{Multiplex Ligation-Dependent Probe Amplification (MLPA) Analyses}

All of the samples that were negative or had a heterozygote mutation for CFTR nextgeneration sequencing were then screened to identify the deletions of the CFTR gene via MLPA (CFTR-SALSA-MLPA-P091, MRC-Holland, Amsterdam, Netherlands) following the manufacturer's recommendations and protocol on ABI 3130XL (Applied Biosystems, Foster City, CA, USA).

The sizes of the exon-specific amplified fragments were identified according to their migration relative to the GeneScan Rox-500 size standard (Applied Biosystems, Foster City, CA, USA) using GeneMapper version 4.0 software. The relative copy numbers of the CFTR gene products were determined using Coffalyser software provided online by the manufacturer (www.mlpa.com). We considered results with values between 0.8 and 1.2 as normal. The relative copy number values above 1.3 were considered duplications, and values below 0.65 were considered deletions. 


\section{Results}

NBS for CF in Turkey has also introduced widespread DNA-based testing for patients. NGS has become the preferred molecular genetics testing method due to its high sensitivity and reliability. It enables multigene sequencing of multiple samples during the same workflow. This provides copious amounts of data and information as compared to conventional methods. This technology, however, requires qualified laboratory scientists and experienced medical geneticists to process and analyze such a large quantity of data.

According to the results, $560(35.1 \%)$ of the 1595 patients carried at least 1 (one) homozygous or heterozygous CF-related variant, while 1035 patients $(64.9 \%)$ had no detected clinically significant variants.

Among the patients who had at least one disease-related variant, 110 had a homozygous mutation for at least one variant $(110 / 560=19.64 \%)$, and 228 patients had two different variants in the compound heterozygous state (40.71\%). Fifty-two of the patients had three variants $(9.3 \%)$; 39 of these 52 had three different mutations in the heterozygous state; 13 patients were homozygous for one mutation and heterozygous for two mutations. One hundred fifty-six (156) patients had one heterozygous mutation (27.86\%); 14 patients had double homozygous mutations (homozygous for two different mutations simultaneously; $2.5 \%$; Figure 1).

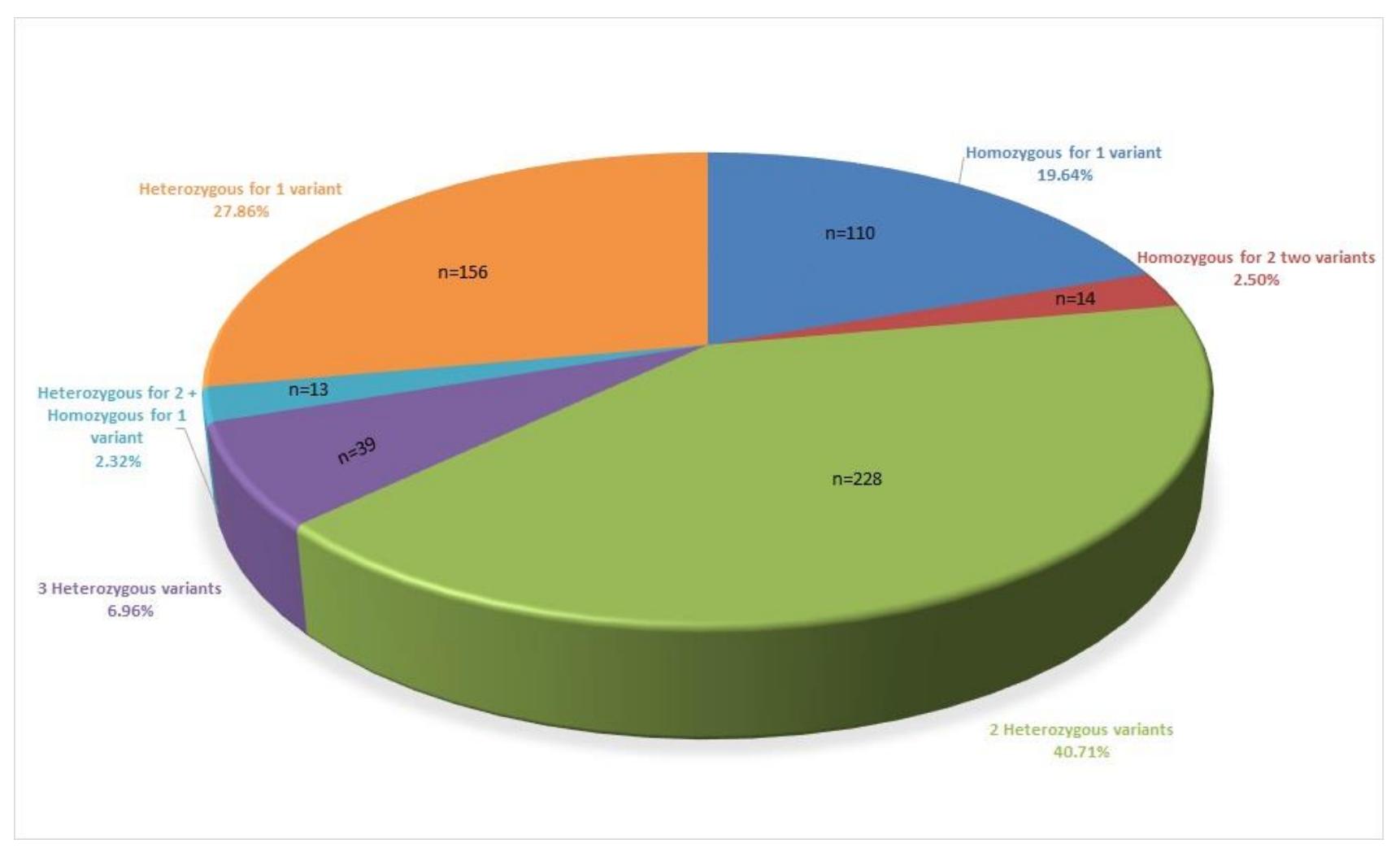

Figure 1. Distribution of patients according to mutation burden.

We detected 15 novel variants that have not been previously reported (Table 1). We found a total of 161 variants in 560 patients with mutations (Table 2). 
Table 1. Detected novel variants and American College of Medical Genetics (ACMG) criteria provided with pathogenicity classification.

\begin{tabular}{|c|c|c|c|}
\hline Variants & Mutation Type & ACMG Classification & Patient Numbers \\
\hline c.2978A > C p.D993A & Missense & $\mathrm{LP}$ & 7 \\
\hline c.1729T > C p.Y577H & Missense & VUS & 2 \\
\hline c. $3468+52 \mathrm{~A}>\mathrm{C}$ & Intronic & VUS & 2 \\
\hline c.3170C > G p.T1057R & Missense & $\mathrm{LP}$ & 1 \\
\hline c. 2982 C > G p.F994L & Missense & VUS & 1 \\
\hline c. $1680-756 \mathrm{C}>\mathrm{T}$ & Intronic & VUS & 1 \\
\hline c. $2054 \mathrm{~A}$ > G p.Q685R & Missense & $\mathrm{LP}$ & 1 \\
\hline c.698T > C p.L233P & Missense & $\mathrm{LP}$ & 1 \\
\hline c.4321delC p.R1438fs*10 & Frameshift & $\mathrm{LP}$ & 1 \\
\hline c. $3468+137 \mathrm{~T}>\mathrm{C}$ & Intronic & VUS & 1 \\
\hline c.7A > T p.R3W & Missense & VUS & 1 \\
\hline c. $1836 \mathrm{~A}>\mathrm{T}$ p.K612N & Missense & LP & 1 \\
\hline c.1217G > C p.G406A & Missense & VUS & 1 \\
\hline c. $53+28 \mathrm{~A}>\mathrm{G}$ & Intronic & VUS & 1 \\
\hline c.3311A > G p.E1104G & Missense & LP & 1 \\
\hline
\end{tabular}

LP: Likely Pathogenic, VUS: Variant of Uncertain Significance.

Table 2. The total list of mutations detected. ${ }^{*}$ Not expected to have a major effect on the disease but have a cumulative effect with other pathogenic variants.

\begin{tabular}{|c|c|c|c|c|}
\hline Variants & Mutation Type & Number of Alleles & ACMG Classification & CFRT2 Database \\
\hline p.V470M & Missense & 399 & Benign * & $\mathrm{N} / \mathrm{A}$ \\
\hline p.L997F & Missense & 66 & $\mathrm{P}$ & Non CF-causing \\
\hline p.P1013L & Missense & 47 & $\mathrm{P}$ & $\mathrm{N} / \mathrm{A}$ \\
\hline p.F508del & Inframe del & 42 & $\mathrm{P}$ & CF-causing \\
\hline p.I148T & Missense & 31 & VUS & Non CF-causing \\
\hline p.F1052V & Missense & 25 & $\mathrm{P}$ & $\begin{array}{l}\text { Varying clinical } \\
\text { consequence }\end{array}$ \\
\hline p.E217G & Missense & 22 & $\mathrm{P}$ & $\mathrm{N} / \mathrm{A}$ \\
\hline p.I807M & Missense & 22 & $\mathrm{P}$ & Non CF-causing \\
\hline c. $2657+5 \mathrm{G}>\mathrm{A}$ & Intronic & 19 & $\mathrm{P}$ & CF-causing \\
\hline c. $2620-15 C>G$ & Intronic & 16 & $\mathrm{P}$ & $\mathrm{N} / \mathrm{A}$ \\
\hline c. $3964-3 C>G$ & Intronic & 16 & $\mathrm{P}$ & $\mathrm{N} / \mathrm{A}$ \\
\hline p.F1052L & Missense & 13 & $\mathrm{P}$ & $\mathrm{N} / \mathrm{A}$ \\
\hline p.K68E & Missense & 13 & $\mathrm{P}$ & $\mathrm{N} / \mathrm{A}$ \\
\hline p.D1312G & Missense & 11 & $\mathrm{P}$ & $\mathrm{N} / \mathrm{A}$ \\
\hline p.D993A & Missense & 9 & LP & $\mathrm{N} / \mathrm{A}$ \\
\hline p.M952I & Missense & 9 & $\mathrm{P}$ & $\mathrm{N} / \mathrm{A}$ \\
\hline p.Y515* & Nonsense & 9 & $\mathrm{P}$ & $\mathrm{N} / \mathrm{A}$ \\
\hline c. $2909-71 G>C$ & Intronic & 8 & $\mathrm{P}$ & $\mathrm{N} / \mathrm{A}$ \\
\hline
\end{tabular}


Table 2. Cont.

\begin{tabular}{|c|c|c|c|c|}
\hline Variants & Mutation Type & Number of Alleles & ACMG Classification & CFRT2 Database \\
\hline c. $3718-2477 \mathrm{C}>\mathrm{T}$ & Intronic & 8 & $\mathrm{P}$ & CF-causing \\
\hline p.D110H & Missense & 8 & $\mathrm{P}$ & CF-causing \\
\hline p.D1152H & Missense & 8 & $\mathrm{P}$ & $\begin{array}{l}\text { Varying clinical } \\
\text { consequence }\end{array}$ \\
\hline p.K684fs*38 & Frameshift & 8 & $\mathrm{P}$ & $\mathrm{N} / \mathrm{A}$ \\
\hline p.M348K & Missense & 8 & $\mathrm{P}$ & $\mathrm{N} / \mathrm{A}$ \\
\hline p.W1282* & Nonsense & 8 & $\mathrm{P}$ & CF-causing \\
\hline p.D806G & Missense & 7 & $\mathrm{P}$ & $\mathrm{N} / \mathrm{A}$ \\
\hline p.G576A & Missense & 7 & VUS & Non CF-causing \\
\hline p.L732* & Nonsense & 7 & $\mathrm{P}$ & CF-causing \\
\hline p.N1303K & Missense & 7 & $\mathrm{P}$ & CF-causing \\
\hline p.R347P & Missense & 7 & $\mathrm{P}$ & CF-causing \\
\hline p.I506V & Missense & 6 & VUS & $\mathrm{N} / \mathrm{A}$ \\
\hline p.L633I & Missense & 6 & $\mathrm{P}$ & $\mathrm{N} / \mathrm{A}$ \\
\hline p.R117C & Missense & 6 & $\mathrm{P}$ & CF-causing \\
\hline p.T388M & Missense & 6 & $\mathrm{P}$ & $\mathrm{N} / \mathrm{A}$ \\
\hline c. $489+3 \mathrm{~A}>\mathrm{G}$ & Intronic & 5 & $\mathrm{P}$ & $\begin{array}{l}\text { Varying clinical } \\
\text { consequence }\end{array}$ \\
\hline p.G542* & Nonsense & 5 & $P$ & $\begin{array}{l}\text { Varying clinical } \\
\text { consequence }\end{array}$ \\
\hline p.R668C & Missense & 5 & $\mathrm{P}$ & Non CF-causing \\
\hline p.R75Q & Missense & 5 & $\mathrm{P}$ & Non CF-causing \\
\hline p.S1235R & Missense & 5 & $\mathrm{P}$ & Non CF-causing \\
\hline p.S877A & Missense & 5 & VUS & $\mathrm{N} / \mathrm{A}$ \\
\hline p.T1220I & Missense & 5 & VUS & $\mathrm{N} / \mathrm{A}$ \\
\hline p.D513G & Missense & 4 & $\mathrm{P}$ & CF-causing \\
\hline p.L1034F & Missense & 4 & $\mathrm{P}$ & $\mathrm{N} / \mathrm{A}$ \\
\hline p.P111L & Missense & 4 & $\mathrm{P}$ & $\mathrm{N} / \mathrm{A}$ \\
\hline p.Q493P & Missense & 4 & $\mathrm{P}$ & $\mathrm{N} / \mathrm{A}$ \\
\hline p.R117H & Missense & 4 & $\mathrm{P}$ & $\begin{array}{l}\text { Varying clinical } \\
\text { consequence }\end{array}$ \\
\hline p.R297Q & Missense & 4 & $\mathrm{P}$ & $\mathrm{N} / \mathrm{A}$ \\
\hline p.W1098C & Missense & 4 & VUS & CF-causing \\
\hline p.A120T & Missense & 3 & $\mathrm{LP}$ & $\begin{array}{l}\text { Varying clinical } \\
\text { consequence }\end{array}$ \\
\hline p.A399V & Missense & 3 & $\mathrm{P}$ & $\mathrm{N} / \mathrm{A}$ \\
\hline p.D836Y & Missense & 3 & $\mathrm{P}$ & Non CF-causing \\
\hline p.E1228G & Missense & 3 & LP & $\mathrm{N} / \mathrm{A}$ \\
\hline p.E528K & Missense & 3 & LP & $\mathrm{N} / \mathrm{A}$ \\
\hline p.E831* & Nonsense & 3 & $\mathrm{P}$ & CF-causing \\
\hline p.E92K & Missense & 3 & $\mathrm{P}$ & CF-causing \\
\hline p.I1234V & Missense & 3 & $\mathrm{P}$ & CF-causing \\
\hline
\end{tabular}


Table 2. Cont.

\begin{tabular}{|c|c|c|c|c|}
\hline Variants & Mutation Type & Number of Alleles & ACMG Classification & CFRT2 Database \\
\hline p.I853I & Synonymous & 3 & VUS & $\mathrm{N} / \mathrm{A}$ \\
\hline p.L183I & Missense & 3 & $\mathrm{P}$ & $\mathrm{N} / \mathrm{A}$ \\
\hline p.M1101R & Missense & 3 & $\mathrm{P}$ & CF-causing \\
\hline p.Q353* & Nonsense & 3 & $\mathrm{P}$ & $\mathrm{N} / \mathrm{A}$ \\
\hline p.R334W & Missense & 3 & $\mathrm{P}$ & CF-causing \\
\hline p.R352Q & Missense & 3 & VUS & CF-causing \\
\hline p.S955A & Missense & 3 & VUS & $\mathrm{N} / \mathrm{A}$ \\
\hline p.V1198M & Missense & 3 & LP & $\mathrm{N} / \mathrm{A}$ \\
\hline c. $1766+3 \mathrm{~A}>\mathrm{G}$ & Intronic & 2 & $\mathrm{P}$ & CF-causing \\
\hline c. $2491-51 \mathrm{~T}>\mathrm{C}$ & Intronic & 2 & VUS & $\mathrm{N} / \mathrm{A}$ \\
\hline c. $3468+52 \mathrm{~A}>\mathrm{C}$ & Intronic & 2 & VUS & $\mathrm{N} / \mathrm{A}$ \\
\hline p.C866T & Missense & 2 & $\mathrm{LP}$ & $\mathrm{N} / \mathrm{A}$ \\
\hline p.E528E & Missense & 2 & $\mathrm{P}$ & $\mathrm{N} / \mathrm{A}$ \\
\hline p.F834L & Missense & 2 & $\mathrm{P}$ & $\mathrm{N} / \mathrm{A}$ \\
\hline p.G1069R & Missense & 2 & $\mathrm{P}$ & $\begin{array}{l}\text { Varying clinical } \\
\text { consequence }\end{array}$ \\
\hline p.I1000fs*2 & Frameshift & 2 & $\mathrm{P}$ & $\mathrm{N} / \mathrm{A}$ \\
\hline p.I1295fs *33 & Frameshift & 2 & $\mathrm{P}$ & $\mathrm{N} / \mathrm{A}$ \\
\hline p.I752S & Missense & 2 & VUS & $\mathrm{N} / \mathrm{A}$ \\
\hline p.K64E & Missense & 2 & $\mathrm{P}$ & $\mathrm{N} / \mathrm{A}$ \\
\hline p.L137fs*15 & Frameshift & 2 & $\mathrm{P}$ & $\mathrm{N} / \mathrm{A}$ \\
\hline p.M952T & Missense & 2 & $\mathrm{P}$ & Unknown significance \\
\hline p.R709* & Nonsense & 2 & $\mathrm{P}$ & CF-causing \\
\hline p.R74W & Missense & 2 & $\mathrm{P}$ & $\begin{array}{l}\text { Varying clinical } \\
\text { consequence }\end{array}$ \\
\hline p.S1373I & Missense & 2 & LP & $\mathrm{N} / \mathrm{A}$ \\
\hline p.T1019A & Missense & 2 & VUS & $\mathrm{N} / \mathrm{A}$ \\
\hline p.T1057A & Missense & 2 & $\mathrm{P}$ & $\mathrm{N} / \mathrm{A}$ \\
\hline p.T1299T & Missense & 2 & VUS & $\mathrm{N} / \mathrm{A}$ \\
\hline p.T966M & Missense & 2 & VUS & $\mathrm{N} / \mathrm{A}$ \\
\hline p.V201M & Missense & 2 & $\mathrm{P}$ & Unknown significance \\
\hline p.V754M & Missense & 2 & $\mathrm{P}$ & Non CF-causing \\
\hline p.Y301C & Missense & 2 & VUS & $\mathrm{N} / \mathrm{A}$ \\
\hline p.Y577H & Missense & 2 & VUS & $\mathrm{N} / \mathrm{A}$ \\
\hline c. $1116+57 \mathrm{C}>\mathrm{G}$ & Intronic & 1 & VUS & $\mathrm{N} / \mathrm{A}$ \\
\hline c. $164+9 \mathrm{~A}>\mathrm{T}$ & Intronic & 1 & VUS & $\mathrm{N} / \mathrm{A}$ \\
\hline c. $1680-756 \mathrm{C}>\mathrm{T}$ & Intronic & 1 & VUS & $\mathrm{N} / \mathrm{A}$ \\
\hline c. $2490+5 \mathrm{G}>\mathrm{T}$ & Intronic & 1 & VUS & $\mathrm{N} / \mathrm{A}$ \\
\hline c. $2909-15 \mathrm{~T}>\mathrm{G}$ & Intronic & 1 & VUS & $\mathrm{N} / \mathrm{A}$ \\
\hline c. $2989-3 C>T$ & Intronic & 1 & VUS & $\mathrm{N} / \mathrm{A}$ \\
\hline c. $3139+80$ delA & Intronic & 1 & VUS & $\mathrm{N} / \mathrm{A}$ \\
\hline c. $3368-4 A>G$ & Intronic & 1 & $\mathrm{P}$ & $\mathrm{N} / \mathrm{A}$ \\
\hline
\end{tabular}


Table 2. Cont.

\begin{tabular}{|c|c|c|c|c|}
\hline Variants & Mutation Type & Number of Alleles & ACMG Classification & CFRT2 Database \\
\hline c. $3468+137 \mathrm{~T}>\mathrm{C}$ & Intronic & 1 & VUS & $\mathrm{N} / \mathrm{A}$ \\
\hline c. $3469-2 A>G$ & Intronic & 1 & $\mathrm{P}$ & $\mathrm{N} / \mathrm{A}$ \\
\hline c. $3963+15 \mathrm{~T}>\mathrm{C}$ & Intronic & 1 & VUS & $\mathrm{N} / \mathrm{A}$ \\
\hline c. $490-165 \mathrm{~T}>\mathrm{C}$ & Intronic & 1 & VUS & $\mathrm{N} / \mathrm{A}$ \\
\hline c. $53+28 \mathrm{~A}>\mathrm{G}$ & Intronic & 1 & VUS & $\mathrm{N} / \mathrm{A}$ \\
\hline c. 870 - 1026delC & Intronic & 1 & VUS & $\mathrm{N} / \mathrm{A}$ \\
\hline p.A1009T & Missense & 1 & $\mathrm{P}$ & $\mathrm{N} / \mathrm{A}$ \\
\hline p.A1113V & Missense & 1 & VUS & $\mathrm{N} / \mathrm{A}$ \\
\hline p.A1364A & Synonymous & 1 & $\mathrm{P}$ & $\mathrm{N} / \mathrm{A}$ \\
\hline p.A455V & Missense & 1 & $\mathrm{P}$ & $\mathrm{N} / \mathrm{A}$ \\
\hline p.D58G & Missense & 1 & $\mathrm{P}$ & $\mathrm{N} / \mathrm{A}$ \\
\hline p.D891G & Missense & 1 & $\mathrm{P}$ & $\mathrm{N} / \mathrm{A}$ \\
\hline p.D924N & Missense & 1 & $\mathrm{P}$ & Unknown significance \\
\hline p.D985E & Missense & 1 & LP & $\mathrm{N} / \mathrm{A}$ \\
\hline p.E1104G & Missense & 1 & LP & $\mathrm{N} / \mathrm{A}$ \\
\hline p.E1409K & Missense & 1 & $\mathrm{P}$ & $\mathrm{N} / \mathrm{A}$ \\
\hline p.E826K & Missense & 1 & $\mathrm{P}$ & $\mathrm{N} / \mathrm{A}$ \\
\hline p.F508C & Missense & 1 & $\mathrm{P}$ & Non CF-causing \\
\hline p.F994C & Missense & 1 & $\mathrm{P}$ & $\mathrm{N} / \mathrm{A}$ \\
\hline p.F994L & Missense & 1 & VUS & $\mathrm{N} / \mathrm{A}$ \\
\hline p.G314A & Missense & 1 & LP & $\mathrm{N} / \mathrm{A}$ \\
\hline p.G406A & Missense & 1 & VUS & $\mathrm{N} / \mathrm{A}$ \\
\hline p.G723D & Missense & 1 & $\mathrm{LP}$ & $\mathrm{N} / \mathrm{A}$ \\
\hline p.I125T & Missense & 1 & $\mathrm{P}$ & $\mathrm{N} / \mathrm{A}$ \\
\hline p.I521F & Missense & 1 & $\mathrm{P}$ & $\mathrm{N} / \mathrm{A}$ \\
\hline p.K1060T & Missense & 1 & $\mathrm{P}$ & $\mathrm{N} / \mathrm{A}$ \\
\hline p.K536E & Missense & 1 & LP & $\mathrm{N} / \mathrm{A}$ \\
\hline p.K612N & Missense & 1 & LP & $\mathrm{N} / \mathrm{A}$ \\
\hline p.K68N & Missense & 1 & VUS & $\mathrm{N} / \mathrm{A}$ \\
\hline p.L1156F & Missense & 1 & VUS & $\mathrm{N} / \mathrm{A}$ \\
\hline p.L227R & Missense & 1 & $\mathrm{P}$ & CF-causing \\
\hline p.L233P & Missense & 1 & LP & $\mathrm{N} / \mathrm{A}$ \\
\hline p.L467F & Missense & 1 & $\mathrm{P}$ & $\mathrm{N} / \mathrm{A}$ \\
\hline p.L568F & Missense & 1 & VUS & $\mathrm{N} / \mathrm{A}$ \\
\hline p.L610I & Missense & 1 & $\mathrm{P}$ & $\mathrm{N} / \mathrm{A}$ \\
\hline p.L88* & Nonsense & 1 & $\mathrm{P}$ & CF-causing \\
\hline p.M1407T & Missense & 1 & $\mathrm{P}$ & $\mathrm{N} / \mathrm{A}$ \\
\hline p.N1432K & Missense & 1 & $\mathrm{P}$ & $\mathrm{N} / \mathrm{A}$ \\
\hline p.N306S & Missense & 1 & VUS & $\mathrm{N} / \mathrm{A}$ \\
\hline p.N417K & Missense & 1 & VUS & $\mathrm{N} / \mathrm{A}$ \\
\hline p.P111P & Synonymous & 1 & VUS & $\mathrm{N} / \mathrm{A}$ \\
\hline
\end{tabular}


Table 2. Cont.

\begin{tabular}{|c|c|c|c|c|}
\hline Variants & Mutation Type & Number of Alleles & ACMG Classification & CFRT2 Database \\
\hline p.P5L & Missense & 1 & $\mathrm{P}$ & $\begin{array}{l}\text { Varying clinical } \\
\text { consequence }\end{array}$ \\
\hline p.Q685R & Missense & 1 & LP & $\mathrm{N} / \mathrm{A}$ \\
\hline p.R1162L & Missense & 1 & $\mathrm{P}$ & Non CF-causing \\
\hline p.R1438fs*10 & Frameshift & 1 & LP & $\mathrm{N} / \mathrm{A}$ \\
\hline p.R31C & Missense & 1 & $\mathrm{P}$ & Non CF-causing \\
\hline p.R334Q & Missense & 1 & $\mathrm{P}$ & $\begin{array}{l}\text { Varying clinical } \\
\text { consequence }\end{array}$ \\
\hline p.R347H & Missense & 1 & $\mathrm{P}$ & CF-causing \\
\hline p.R3W & Missense & 1 & VUS & $\mathrm{N} / \mathrm{A}$ \\
\hline p.R668L & Missense & 1 & $\mathrm{P}$ & $\mathrm{N} / \mathrm{A}$ \\
\hline p.R785* & Nonsense & 1 & $\mathrm{P}$ & CF-causing \\
\hline p.R997F & Missense & 1 & $\mathrm{P}$ & $\mathrm{N} / \mathrm{A}$ \\
\hline p.S1426P & Missense & 1 & $\mathrm{P}$ & $\mathrm{N} / \mathrm{A}$ \\
\hline p.S307N & Missense & 1 & $\mathrm{P}$ & $\mathrm{N} / \mathrm{A}$ \\
\hline p.S912L & Missense & 1 & $\mathrm{P}$ & Unknown significance \\
\hline p.S945L & Missense & 1 & $\mathrm{P}$ & CF-causing \\
\hline p.T1057R & Missense & 1 & LP & $\mathrm{N} / \mathrm{A}$ \\
\hline p.T351S & Missense & 1 & $\mathrm{P}$ & $\mathrm{N} / \mathrm{A}$ \\
\hline p.T465N & Missense & 1 & $\mathrm{P}$ & $\mathrm{N} / \mathrm{A}$ \\
\hline p.T760M & Missense & 1 & $\mathrm{P}$ & $\mathrm{N} / \mathrm{A}$ \\
\hline p.V855I & Missense & 1 & VUS & $\mathrm{N} / \mathrm{A}$ \\
\hline p.V920L & Missense & 1 & $\mathrm{P}$ & $\mathrm{N} / \mathrm{A}$ \\
\hline p.V938G & Missense & 1 & LP & $\mathrm{N} / \mathrm{A}$ \\
\hline p.W1098L & Missense & 1 & $\mathrm{P}$ & $\mathrm{N} / \mathrm{A}$ \\
\hline p.Y424Y & Synonymous & 1 & $\mathrm{P}$ & $\mathrm{N} / \mathrm{A}$ \\
\hline p.Y919C & Missense & 1 & $\mathrm{P}$ & $\mathrm{N} / \mathrm{A}$ \\
\hline
\end{tabular}

P: Pathogenic, LP: Likely Pathogenic, VUS: Variant of Uncertain Significance, N/A: not available.

The most frequent variant was p.V470M; 313 of 560 patients had this variant. p.V470M is classified as a polymorphism according to ACMG, and it is known to have a cumulative effect when combined with other mutations [13]. Therefore, this mutation was reported only when the patient had other clinically significant mutations (figures were prepared excluding p.V470M). Thus, no other common or benign variants were reported other than p.V470M. The most frequent pathogenic mutation, p.L997F, was detected in 61 of 560 patients. The second most frequent pathogenic variant was p.P1013L, and it was detected in 44 of 560 patients. p.F508del was the third most frequent mutation, observed in 39 of 560 patients (Figure 2). 


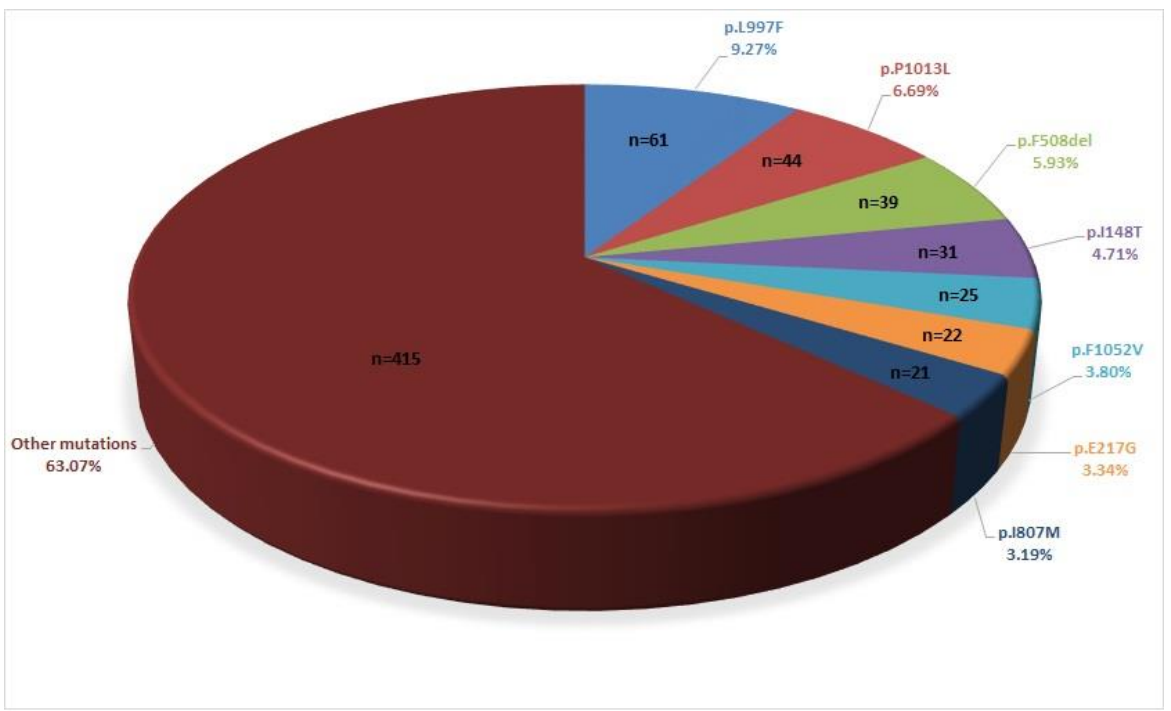

Figure 2. Distribution of the most frequent mutations.

The vast majority of the mutant alleles were single base-pair substitutions; 118 of the 160 variants were missense mutations. Twenty-three of the patients had an intronic variant, while nine patients had a nonsense mutation (calculations were made excluding p.V470M; Figure 3).

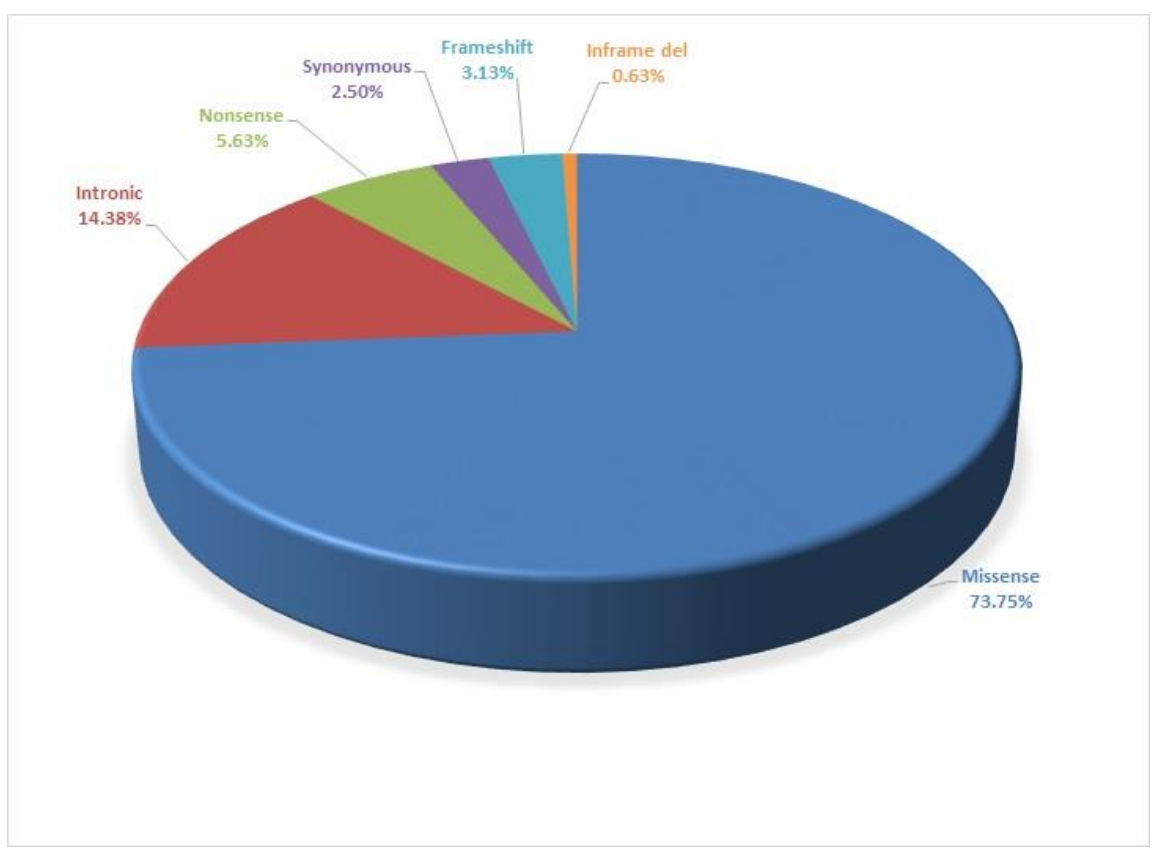

Figure 3. Description of the detected types of mutations.

Deletion-duplication of CFTR was investigated with the MLPA method in 391 patients with no mutations, and deletion was detected in 3 patients. Two of three patients had exon 10 deletions, and the others had multiple exon (exon 4-11) deletion-these patients had no mutations detected by sequencing (MLPA results are not shown in tables and figures).

\section{Discussion}

Newborn genetic screening has been a remarkable achievement as a public health intervention, providing population-wide detection of disorders that have greatly improved the lives of thousands of affected children and even more than a million newborns each year. 
However, both the economic and social forces pose significant ethical and clinical challenges to NBS, and they mainly regard accommodating laboratory and clinical standards to rapid developments and preparing health systems to respond to such advances.

With the trend of increasing molecular test requests, all genetic testing laboratories have been forced to adapt to the times and utilize technology with high sensitivity and reliability. As a result of the CF NBS program, due to the high number of samples that require quick and trustworthy results, next-generation sequencing has become a first-tier testing method, largely replacing targeted sequencing or other conventional methodologies. However, even with powerful test technologies such as NGS, there remains a need to multiplex such techniques, as conducted in this study with the combination of NGS and MLPA used to identify newborns or children with anomalies that may-or may notlead to disease. Furthermore, if these NBS programs evolve and include more than a single disease testing method, they may also be able to find children for whom treatment is available.

In regard to CF, more than 2000 mutations have been identified in the CFTR gene, which may lead to a loss of function of this anion channel at the apical plasma membrane of secretory epithelia [14]. The majority of all of these variants are point mutations or other small sequence changes, and up to $2 \%$ of $\mathrm{CF}$ alleles are likely gene rearrangements, including large deletions, insertions, and duplications [15]. However, we detected large deletions in only 3 patients (two heterozygous and one homozygous, a total of four deleted alleles) among the 391 patients on whom MLPA analysis was conducted. This accounts for $0.5 \%$ of 391 patients (782 alleles), which is a lower figure than that in published cohort studies.

The most common CFTR mutation, p.F508del (delF508 by legacy nomenclature), accounts for approximately $66-70 \%$ of identified mutant alleles worldwide $[16,17]$ while it was observed in only $39(6 \%)$ patients with at least one mutation, making it the third most frequent mutation in our study. Even though the p.F508del mutation accounts for $70 \%$ of CF mutations in white patients of northern European descent, it has differing percentages in other populations [18]. In the Russian population, the p.F508del mutation accounts for $53 \%$ of mutations [19], and this is caused by the specific ethnic background of the population. Thus, screening for known $\mathrm{CF}$ gene mutations did not help to ameliorate such inequities in our population, but the combination of NGS with MLPA was more successful, as explained in the Results Section. NGS has dramatically advanced the process of genetic variant identification; however, clinical interpretation remains a challenge. Even though the largest publicly-available population datasets for $\mathrm{CF}$ to date are gnomAD and CFTR2, participants are classified into seven different ethnic groups without clustering any other major populations, which are most likely of mixed backgrounds, especially in Mediterranean countries.

The most frequent mutation in the Turkish population according to this study, in which all of the patients enrolled were selected by the NBS program, is p.L997F. Thus, this study provides important data to clarify the complexity of this variant in relation to the disease [20]. L997F was identified by Lucarelli et.al. who reported that it leads to mild CF or cystic fibrosis-related disorders (CFRD) [21]. In other reports, this variant was associated with pancreatitis [22,23], CFRD, and mild CF [24]. There are publications stating that this variant has no relation with $\mathrm{CF}$ disease, and there are also publications opposing the notion that it is causative $[21,25]$. Since the false-negative rate of NBS is at least $8.7 \%$, depending on the method, patients with this mutation who receive false negative NBS results have an increased risk of presentation with meconium ileus after birth or dehydration and pancreatitis at a later age [26].

Similarly, p.I148T is classified as VUS according to the ACMG criteria, with publicly open web-based datasets from VARSOME showing evidence-based data of its relation to CF. Moreover, several studies also suggest that it might have a cumulative effect on CF and/or the CFRD phenotype with congenital agenesis of vas deferens perpetuation when identified with other well-known pathogenic variants in different populations $[24,27,28]$. 
In contrast with the results of our study, it has been suggested in some studies that while the effect of the V470M variant is classified as benign according to the ACMG criteria, it is clinically significant when it is combined with other pathogenic variants [13,29]. Thus, it is only shown in Table 2, displaying the variant distribution. There is also a possibility that some variants such as V470M at a higher frequency may act as disease modifiers rather than casual variants; therefore, it is important for phenotype interpretation. There is also a chance that the CF genotype prevalence of V470M in gnomAD might be inflated since the analyses were allele based. However, this requires further investigations involving deep phenotyping and prospective phenotype correlation analysis, which our center intends to conduct in future studies.

In recent years, a considerable effort was focused on molecular therapies that can directly interact with CFTR mutants has indicated the importance of identifying the mutations of CFTR and the complexity of CFTR mutant phenotypes at the cellular levels $[10,30,31]$. Moreover, early diagnosis through NBS is the best way to prevent primary and secondary manifestations of the disease.

In populations such as those in the Mediterranean region, consanguineous marriage makes the incidence of autosomal recessive diseases such as CF in this study higher. Most interestingly, we reported double homozygous CFTR mutation in transposition status among patients whose parents were first-degree cousins, and their clinical picture was severe. Double homozygous CFTR mutations are a very rare phenomenon, but they are reported more commonly in the Saudi population due to consanguinity [32]. Thus, more precautions and family counseling to increase awareness about the risk of such relationships should be conducted to prevent such extremely rare phenomena. Genetic counseling and medical genetic assessment, together with the diagnostic approach, are becoming increasingly important. Advances in medical genetics and testing technology permit the diagnosis of ever more diseases but also compel society to reconsider how NBS as a public health measure may best serve children, their families, and their communities.

NBS for CF as a public health program in Turkey has achieved enormous success in regard to genetic testing to the extent that it may be implemented in other areas. Moreover, the most crucial step for effective CF management is the early and accurate diagnosis, as provided by this program, and our datasets are available to other centers for variant interpretation.

Author Contributions: S.T.B. and A.B. formed the aim, planned and designed the research, and planned the experiments. C.M. performed data curation. D.O. and D.U.A. evaluated the patients. I.B., O.S. and C.M. performed the experiments with the NGS method. C.R. and A.H. performed experiments with the MLPA method. S.T.B., C.R. and A.H. performed MLPA analysis. A.B., I.B., O.S. and C.M. performed bioinformatics analysis. S.T.B., A.B. and C.M. wrote the original draft. S.T.B. and A.B. reviewed and edited the original draft. All authors have read and agreed to the published version of the manuscript.

Funding: This research received no external funding.

Institutional Review Board Statement: The research was reviewed and approved by the Cukurova University Institutional Ethics Committee (65/02.06.17 and 68/08.09.2017) in compliance with the Declaration of Helsinki 11, and informed parental consent was received from all the participants before inclusion.

Informed Consent Statement: Informed consent was obtained from all patients or from their parents enrolled in the study.

Data Availability Statement: The datasets used and/or analysed during the current study are avalible from the corresponding author upon reasonable request.

Acknowledgments: All participants were informed of and signed consent/permission for this research in accordance with the Declaration of Helsinki. The parental consent form was obtained from the children included in this study. Ethics approval was received from the Cukurova University Ethical Committee. The datasets used and/or analyzed during the current study are available from 
the corresponding author upon reasonable request. We would like to thank the editorial board of "MDPI" for their review.

Conflicts of Interest: The authors declare that they have no conflict of interests.

\section{References}

1. Schrijver, I. Mutation Distribution in Expanded Screening for Cystic Fibrosis: Making Up the Balance in a Context of Ethnic Diversity. Clin. Chem. 2011, 57, 799-801. [CrossRef] [PubMed]

2. World Health Organization. The Molecular Genetic Epidemiology of Cystic Fibrosis. Available online: https://www.who.int/ genomics/publications/en/HGN_WB_04.02_report.pdf?ua=1 (accessed on 13 December 2020).

3. Lago, J.E.F.; Cayarga, A.A.; González, Y.J.G.; Mesa, T.C. A simple, fast and inexpensive method for mutation scanning of CFTR gene. BMC Med. Genet. 2017, 18, 58. [CrossRef] [PubMed]

4. Rommens, J.M.; Iannuzzi, M.C.; Kerem, B.; Drumm, M.L.; Melmer, G.; Dean, M.; Rozmahel, R.; Cole, J.L.; Kennedy, D.; Hidaka, N.; et al. Identification of the cystic fibrosis gene: Chromosome walking and jumping. Science 1989, 245, 1059-1065. [CrossRef] [PubMed]

5. Wang, Y.; Wrennall, J.A.; Cai, Z.; Li, H.; Sheppard, D.N. Understanding how cystic fibrosis mutations disrupt CFTR function: From single molecules to animal models. Int. J. Biochem. Cell Biol. 2014, 52, 47-57. [CrossRef]

6. Human Gene Mutation Database. Available online: http://www.hgmd.cf.ac.uk/ac/gene.php?gene=CFTR (accessed on 13 December 2020).

7. Svensson, A.M.; Chou, L.S.; Miller, C.E.; Robles, J.A.; Swensen, J.J.; Voelkerding, K.V.; Mao, R.; Lyon, E. Detection of large rearrangements in the cystic fibrosis transmembrane conductance regulator gene by multiplex ligation-dependent probe amplification assay when sequencing fails to detect two disease-causing mutations. Genet. Test. Mol. Biomark. 2010, 14, 171-174. [CrossRef]

8. Mall, M.A.; Hartl, D. CFTR: Cystic fibrosis and beyond. Eur. Respir. J. 2014, 44, 1042. [CrossRef]

9. Cystic Fibrosis Foundation. Cystic Fibrosis Foundation Patient Registry 2018 Annual Data Report. @2020; Cystic Fibrosis Foundation: Bethesda, MD, USA, 2019.

10. De Boeck, K.; Zolin, A.; Cuppens, H.; Olesen, H.V.; Viviani, L. The relative frequency of CFTR mutation classes in European patients with cystic fibrosis. J. Cyst. Fibros. Off. J. Eur. Cyst. Fibros. Soc. 2014, 13, 403-409. [CrossRef]

11. TÜIK. Available online: https://data.tuik.gov.tr/Bulten/Index?p=Dogum-Istatistikleri-2019-33706 (accessed on 14 December 2020).

12. Şaşihüseyinoğlu, A.; Altıntaş, D.U.; Bişgin, A.; Doğruel, D.; Yılmaz, M.; Serbes, M. Two years of newborn screening for cystic fibrosis in Turkey: Çukurova experience. Turk. J. Pediatrics 2019, 61, 505-512. [CrossRef]

13. Nefzi, M.; Hadj Fredj, S.; Tebib, N.; Barsaoui, S.; Boussetta, K.; Siala, H.; Messaoud, T. Contribution of M470V variant to cystic fibrosis: First study in CF and normal Tunisian population. Pathol. Biol. 2015, 63, 169-174. [CrossRef]

14. Cutting, G.R. Cystic fibrosis genetics: From molecular understanding to clinical application. Nat. Rev. Genet. 2015, 16, 45-56. [CrossRef]

15. Dequeker, E.; Stuhrmann, M.; Morris, M.A.; Casals, T.; Castellani, C.; Claustres, M.; Cuppens, H.; des Georges, M.; Ferec, C.; Macek, M.; et al. Best practice guidelines for molecular genetic diagnosis of cystic fibrosis and CFTR-related disorders-Updated European recommendations. Eur. J. Hum. Genet. 2009, 17, 51-65. [CrossRef] [PubMed]

16. Bobadilla, J.L.; Macek, M., Jr.; Fine, J.P.; Farrell, P.M. Cystic fibrosis: A worldwide analysis of CFTR mutations-Correlation with incidence data and application to screening. Hum. Mutat. 2002, 19, 575-606. [CrossRef] [PubMed]

17. Petrova, G.; Yaneva, N.; Hrbková, J.; Libik, M.; Savov, A.; Macek, M., Jr. Identification of 99\% of CFTR gene mutations in Bulgarian-, Bulgarian Turk-, and Roma cystic fibrosis patients. Mol. Genet. Genom. Med. 2019, 7, e696. [CrossRef]

18. American College of Obstetricians and Gynecologists; American College of Medical Genetics. Preconception and Prenatal Carrier Screening for Cystic Fibrosis: Clinical and Laboratory Guidelines; American College of Obstetricians and Gynecologists: Washington, DC, USA; American College of Medical Genetics: Bethesda, MD, USA, 2001.

19. Petrova, N.V.; Kashirskaya, N.Y.; Vasilyeva, T.A.; Kondratyeva, E.I.; Zhekaite, E.K.; Voronkova, A.Y.; Sherman, V.D.; Galkina, V.A.; Ginter, E.K.; Kutsev, S.I.; et al. Analysis of CFTR Mutation Spectrum in Ethnic Russian Cystic Fibrosis Patients. Genes 2020, 11, 554. [CrossRef] [PubMed]

20. Strom, C.M.; Redman, J.B.; Peng, M. The dangers of including nonclassical cystic fibrosis variants in population-based screening panels: P.L997F, further genotype/phenotype correlation data. Genet. Med. Off. J. Am. Coll. Med. Genet. 2011, 13, 1042-1044. [CrossRef] [PubMed]

21. Lucarelli, M.; Narzi, L.; Pierandrei, S.; Bruno, S.M.; Stamato, A.; d'Avanzo, M.; Strom, R.; Quattrucci, S. A new complex allele of the CFTR gene partially explains the variable phenotype of the L997F mutation. Genet. Med. Off. J. Am. Coll. Med. Genet. 2010, 12, 548-555. [CrossRef] [PubMed]

22. LaRusch, J.; Jung, J.; General, I.J.; Lewis, M.D.; Park, H.W.; Brand, R.E.; Gelrud, A.; Anderson, M.A.; Banks, P.A.; Conwell, D.; et al. Mechanisms of CFTR functional variants that impair regulated bicarbonate permeation and increase risk for pancreatitis but not for cystic fibrosis. PLoS Genet 2014, 10, e1004376. [CrossRef] [PubMed]

23. Sofia, V.M.; Surace, C.; Terlizzi, V.; Da Sacco, L.; Alghisi, F.; Angiolillo, A.; Braggion, C.; Cirilli, N.; Colombo, C.; Di Lullo, A.; et al. Trans-heterozygosity for mutations enhances the risk of recurrent/chronic pancreatitis in patients with Cystic Fibrosis. Mol. Med. 2018, 24, 38. [CrossRef] [PubMed] 
24. Terlizzi, V.; Castaldo, G.; Salvatore, D.; Lucarelli, M.; Raia, V.; Angioni, A.; Carnovale, V.; Cirilli, N.; Casciaro, R.; Colombo, C.; et al. Genotype-phenotype correlation and functional studies in patients with cystic fibrosis bearing CFTR complex alleles. J. Med. Genet. 2017, 54, 224-235. [CrossRef]

25. Derichs, N.; Schuster, A.; Grund, I.; Ernsting, A.; Stolpe, C.; Körtge-Jung, S.; Gallati, S.; Stuhrmann, M.; Kozlowski, P.; Ballmann, M. Homozygosity for L997F in a child with normal clinical and chloride secretory phenotype provides evidence that this cystic fibrosis transmembrane conductance regulator mutation does not cause cystic fibrosis. Clin. Genet. 2005, 67, 529-531. [CrossRef]

26. Taccetti, G.; Botti, M.; Terlizzi, V.; Cavicchi, M.C.; Neri, A.S.; Galici, V.; Mergni, G.; Centrone, C.; Peroni, D.G.; Festini, F. Clinical and Genotypical Features of False-Negative Patients in 26 Years of Cystic Fibrosis Neonatal Screening in Tuscany, Italy. Diagnostics 2020, 10, 446. [CrossRef] [PubMed]

27. Suaud, L.; Yan, W.; Rubenstein, R.C. Abnormal regulatory interactions of I148T-CFTR and the epithelial Na+ channel in Xenopus oocytes. Am. J. Physiol. Cell Physiol. 2007, 292, C603-C611. [CrossRef] [PubMed]

28. Monaghan, K.G.; Highsmith, W.E.; Amos, J.; Pratt, V.M.; Roa, B.; Friez, M.; Pike-Buchanan, L.L.; Buyse, I.M.; Redman, J.B.; Strom, C.M.; et al. Genotype-phenotype correlation and frequency of the 3199del6 cystic fibrosis mutation among I148T carriers: Results from a collaborative study. Genet. Med. Off. J. Am. Coll. Med. Genet. 2004, 6, 421-425. [CrossRef] [PubMed]

29. Loumi, O.; Cuppens, H.; Bakour, R.; Benabadji, M.; Baghriche, M.; Marynen, P.; Cassiman, J.J. An Algerian child homozygous for the M470V polymorphism and for a deletion of two nucleotides in exon 10 of the CFTR gene, shows severe cystic fibrosis symptoms. Genet. Couns. 1992, 3, 205-207. [PubMed]

30. Veit, G.; Da Fonte, D.F.; Avramescu, R.G.; Premchandar, A.; Bagdany, M.; Xu, H.; Bensinger, D.; Stubba, D.; Schmidt, B.; Matouk, E.; et al. Mutation-specific dual potentiators maximize rescue of CFTR gating mutants. J. Cyst. Fibros. Off. J. Eur. Cyst. Fibros. Soc. 2020, 19, 236-244. [CrossRef] [PubMed]

31. Veit, G.; Avramescu, R.G.; Chiang, A.N.; Houck, S.A.; Cai, Z.; Peters, K.W.; Hong, J.S.; Pollard, H.B.; Guggino, W.B.; Balch, W.E.; et al. From CFTR biology toward combinatorial pharmacotherapy: Expanded classification of cystic fibrosis mutations. Mol. Biol. Cell 2016, 27, 424-433. [CrossRef] [PubMed]

32. Banjar, H.H.; Tuleimat, L.; El Seoudi, A.A.A.; Mogarri, I.; Alhaider, S.; Nizami, I.Y.; AlMaghamsi, T.; Alkaf, S.A.; Moghrabi, N. Genotype patterns for mutations of the cystic fibrosis transmembrane conductance regulator gene: A retrospective descriptive study from Saudi Arabia. Ann. Saudi Med. 2020, 40, 15-24. [CrossRef] 